\title{
Differential Dose Distribution in Transition from 3-D CRT to IMRT Treatment Plans for Glioblastoma Multiforme' at Stage III or IV: A Clinical Study of a Brain Tumor
}

Sadiq R. Malik, Shohel Reza, M.M Shakhawat Hossain

Radiation Oncology Division, Delta Hospital Ltd.

Correspondence Address: Sadiq R. Malik, Chief Radiation Oncology Physicist, Delta Med. College \& Hospital, Mirpur-1,

Dhaka-1216, Bangladesh, EMAIL: smalik51@hotmail.com

\begin{abstract}
Advancement in Cancer Therapy Technology (CTT) due to Software, Hardware and precise delivery of radiation dose has enhanced the quality of life of cancer patients. This report aims at the application of 3-D CRT (Three Dimensional Conformal Radiation Therapy) and IMRT (Intensity Modulated Radiation Therapy) for a quality of treatment. Other anatomical sites viz. Prostate, Lung, etc. may also be treated provided a better tool is applied for target delineation for which FUSION of CT and MRI images are used to ascertain differences in tissue density. This Fusion image of $3 \mathbf{~ m m}$ slices offer accurate contouring of the tumor. The oncologist and/or physicist perform delineation of (I) GTV (Gross Tumor Volume), (II) CTV (Clinical Target Volume), (III) PTV (Planning Target Volume), (IV) TV (Treated Volume) and (V) OARs (Organs at Risk). This is done to secure conformal dose distribution and justify the clinical objectives of Tumor Control Probability (TCP) by reducing the normal tissue complication probability (NTCP).

The implication of this study outlines the fundamental goal of effective treatment procedures by comparing treatment plans of 3-D CRT and IMRT. Tolerance levels of dose to different organs are optimized by the analysis of random and systemic geometrical deviations, margin on target volumes, conformity index (CI), patient selection process and, of course, the shape and stage of target. The comparative parameters of treatment plans are segmented and tabulated to implicate the application of necessary tools to decide on a treatment plan for similar patients. Key word: 3DCRT, IMRT, GBM, PTV, CTV, GTV
\end{abstract}

\section{INTRODUCTION}

3-D CRT means those treatments that are based on 3-D anatomic information and use the dose distribution (1, 2 ) that conform as closely as possible to the target volume and minimum possible dose to to normal tissue maximizing tumor control probability
(TCP). 3-D CRT encompasses the physical and biologic rationales in achieving the desired clinical results (3). The target delineation, therefore, migrates from GTV to CTV, PTV and TV (treated volume) as the margins are incorporated to cover PTV adequately within $95-105 \%$ isodose surface. IMRT is developed using MLC to define the beam intensity independently in different regions of each incident beam to produce uniform dose to the target volume. The position of the leaves of MLC can be varied in time with a fixed or moving gantry. Techniques in IMRT could be STEP and SHOOT, Dynamic MLC or Tomotherapy.

Comparative analysis is essential in selecting one or the other procedures in treating a cancer patient. The anatomic site is selected, in this report, to illustrate the potential of treatment plans in treating such a CANCER.

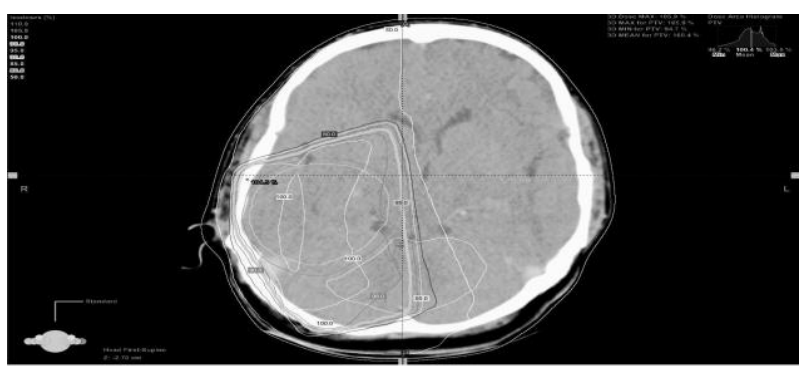

Figure1: (a) 95\% isodose line (green) in a 3D plan

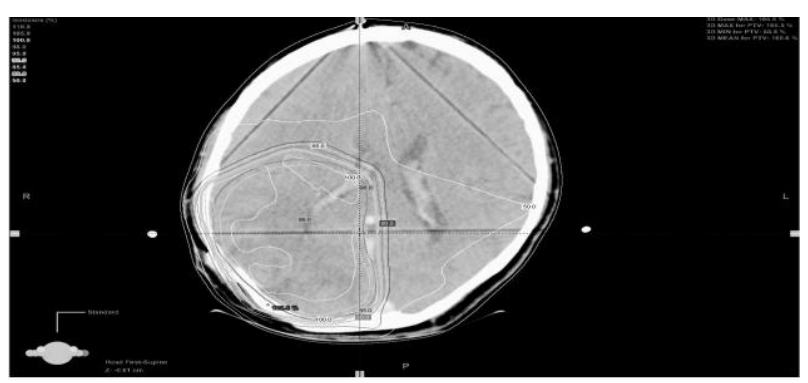

Figure 1: (b) $95 \%$ isodose line (green) in an IMRT plan 


\section{MATERIAL AND METHODS}

\subsection{Concept of the treatment plan}

For Brain (Glomma, Glioblastoma Mutiforme, Astrocytoma, Meningioma, etc.) where $\max$ tolerance dose of Brain Stem (54 Gy), Optic Nerve (54 Gy), Trigeminal Nerve (13 Gy) (4), are taken into consideration in developing a treatment plan. 3D versus IMRT plans are assessed and evaluated and tabulated (Table 1) to express the merit of each one.

Glioblastoma or glioblastoma multiforme' ("multiforme" is no longer part of the WHO designation, though glioblastoma is still often abbreviated as "GBM") is the highest grade gliomma (grade IV) tumor, is the most malignant form of astrocytoma, and is synonymous with a grade IV glioma. The histologic features that distinguish glioblastoma from all other grades are the presence of necrosis (dead cells) and increase of blood vessels around the tumor. Grade IV tumors are always rapidly growing and highly malignant tumors (5).

\subsection{Measurement Tools}

FUSION of CT and MRI images are transferred to Planning Computer and LAND MARKS (fiducials) are selected to register the images. The procedures for imaging for Treatment planning start from CT scanner, with immobilization devices, which are customized for the patient. The GTV is delineated at the contrastenhancing edge of the tumour (not oedema) on postsurgical gadolinium enhanced T1-weighted MRI scans fused with planning CT (6). Contouring of all volumes is drawn and required margins are applied as per protocol (2). Software for planning is then applied with required beam energies and gantry angle selection and MLC activation. A satisfactory treatment plan is approved by evaluating all the parameters for 3-D and IMRT plans where DVH, hotspots and avoidance of OAR are examined. Criteria to approve the plan are tabulated in table 1 .

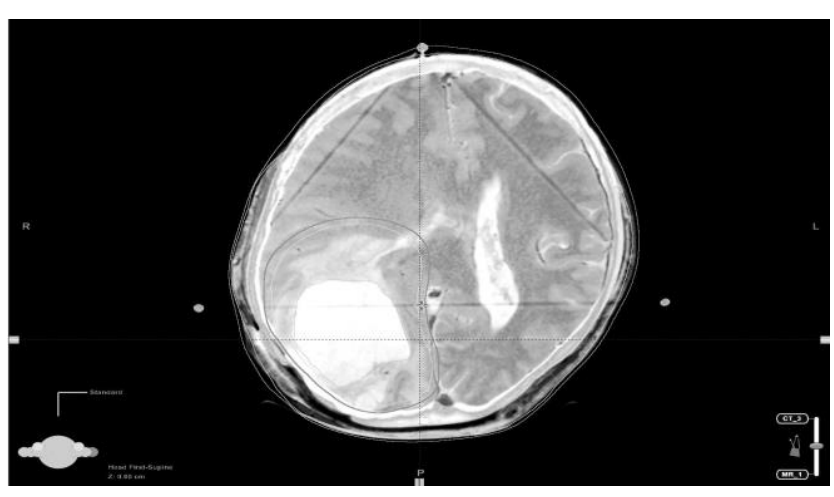

Figure 2.Target when FUSED (CT \& MRI) showing GLIOMA (white mass).

\subsubsection{IMRT: Components for Treatment Delivery}

a. CT Simulator b. Eclipse Tx Planning System for 3DCRT c. Inverse Planning Software for IMRT d. Record and Verification System e. CLINAC linear accelerator; Millennium MLC, Exact Couch and Portal Vision

\subsubsection{CASE Presentation}

i. Patient Selection, ii. Immobilization, iii. Treatment Delivery, iv. Portal Imaging, v. Not obese (entire external contour visualized in CT scan, VI. Patients in pain and in co-operative, vii. Irregularly-shaped tumors, viii. Tumors that wrap around healthy organs, ix. Tumors that are nearby critical normal structures, x. $3 \mathrm{~mm}$ slice thickness, or less, for high quality DRRs. and xi. Intravenous contrast: for soft tissue or lymph nodes (7).

\subsubsection{Anatomy}

a. Anatomical structures contoured by Physician and Physicist b. 3D rendering of Target and Critical Structures

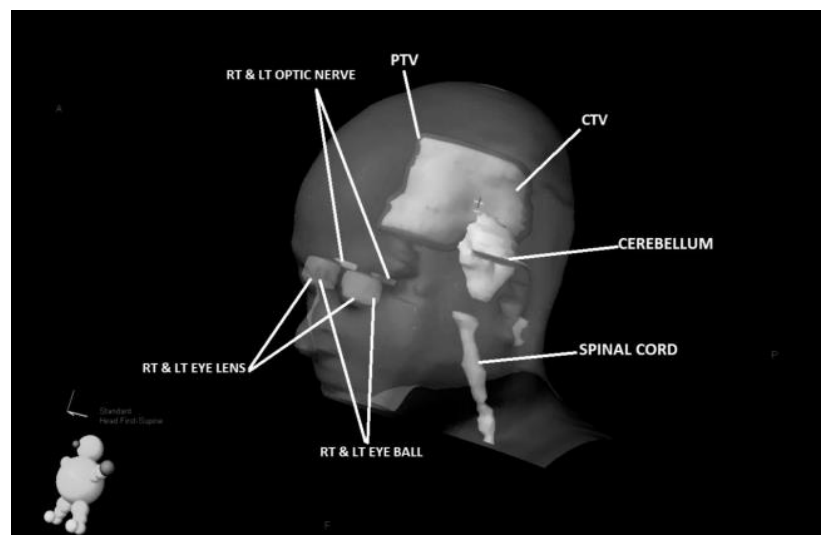

Fig. 3: 3-D structure showing Tumor and a few Organs at Risk 


\subsubsection{Planning Goals}

i. Dose/fraction; Total Dose

ii. Dose limit of Critical Structures

iii. Spinal Cord (45 Gy), Brain Stem (54 Gy) (8), etc.

iv. Gantry Positions (Field numbers 5,7,9 are equally Spaced and non overlapping fields)

v. Dose Constraints: Head and Neck

vi. Inverse Planning for IMRT and Forward Planning for 3D CRT

vii. Isodose Distributions

viii. Plan Review: Start with DVH: Examine Target Dose Heterogeneity, Dose to Critical Structures, isodose distribution, hot and cold spots

ix. Record and Verify system: Approved plan Transfer, Patient Safety

x. QA of Plan: EPID

xi. DRRs for setup verification

xii. Independent Check for SETUP: First Day Verification before treatment

xiii. IMRT is a process. 3D CRT also requires most of these QA

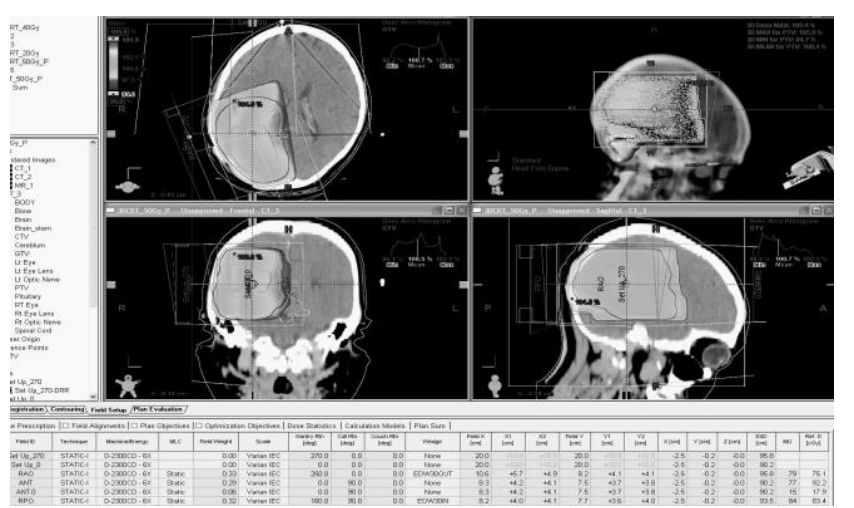

Figure 4 (a) Beam arrangement for 3DCRT plan

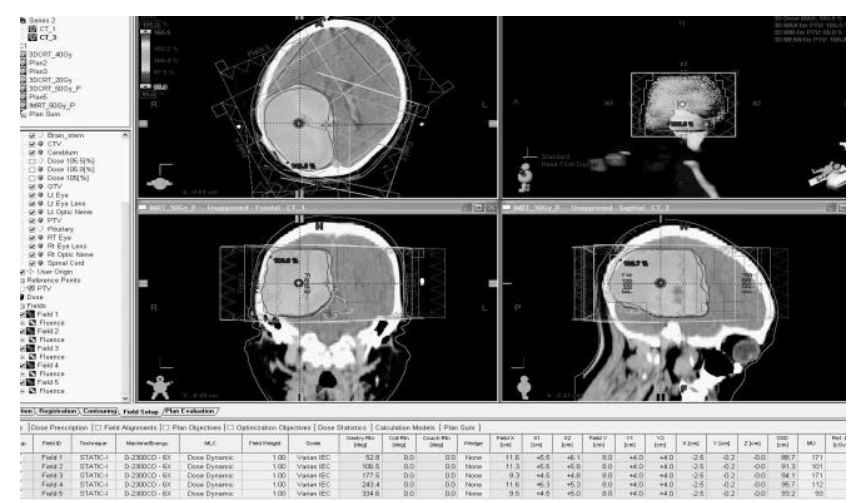

Figure 4 (b) Beam arrangement for IMRT

Figure 4 (a) and 4 (b) show four panes of treatment plans with ISODOSES and Organ Contours for 3-D showing hot spot, coverage, etc.

\section{RESULTS AND DISCUSSION}

3-D and IMRT Treatment Plans are completed with necessary application of tools like Beam Energy $(6 \mathrm{MeV})$, beam angles, number of beams, collimator setting, wedge setting when needed in 3-D, shielding of OAR by MLC leaf manipulation, $\mathrm{DVH}$, tolerance dose to critical organs with $\%$ of volume dose to each organ, etc.
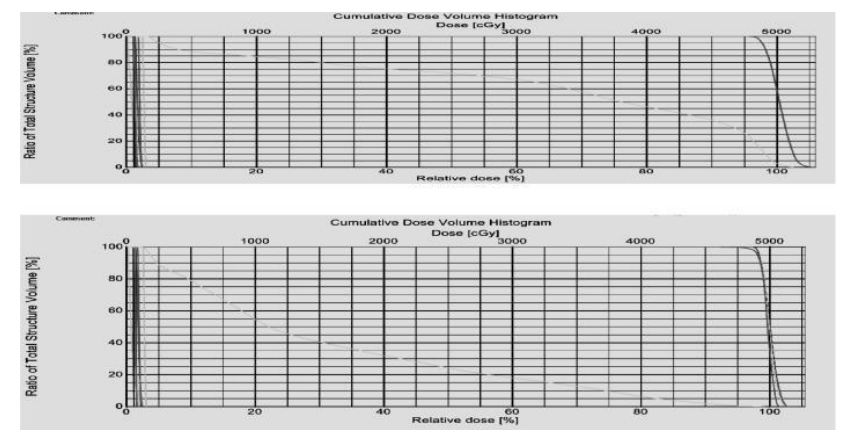

Figure 5. (a) DVH for 3-D (upper) and IMRT (lower)

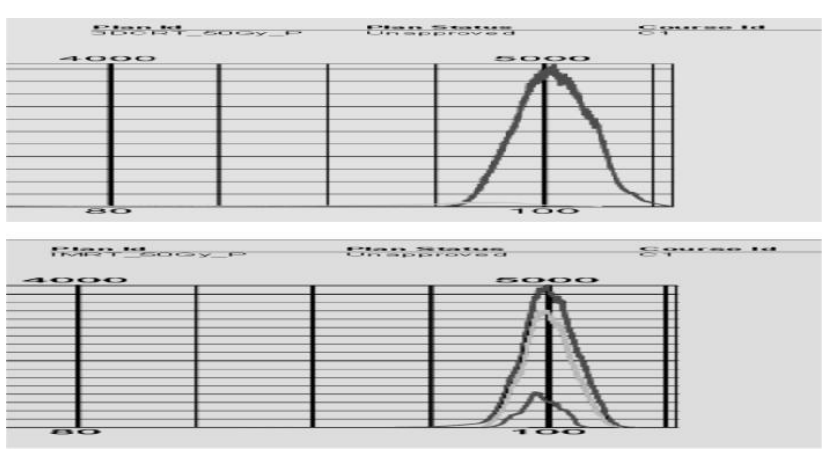

Figure 5 (b) Differential DVH for both plans

Table 1: Estimated parameters considered before approval of the Tx Plan in each case

\begin{tabular}{|c|c|}
\hline 3-DCRT & IMIR'T \\
\hline $\begin{array}{l}\text { Volume of PTV }=235.92 \mathrm{~cm}^{3} \\
\text { Volume of } \mathrm{CTV}=176.85 \mathrm{~cm}^{3} \\
\text { Volume of } \mathrm{GTV}=29.11 \mathrm{~cm}^{3}\end{array}$ & Same as the 3DCir I Volume \\
\hline Prescribed Dose, $2 \mathrm{~Gy} \times 25 \mathrm{fx}=50 \mathrm{~Gy}$ & Prescribed Dose, $2 \mathrm{~Gy} \times 25 \mathrm{fx}=50$ Gy \\
\hline Isodose Normalized by $100 \%$ & Isodose Normalized by $99,0 \%$ \\
\hline Homogeneous Distribution & Heterogeneous Distribution \\
\hline $\begin{array}{l}\text { Organ At Risk (OAR) dose: } \\
\text { a. Rt optic nerve }=135 \mathrm{cGy} \\
\text { b. Ll oplic nerve }=102 \mathrm{cGy} \\
\text { c. Rt cye lens }=69 \mathrm{cGy} \\
\text { d. L1 cye lens }=57 \mathrm{cGy} \\
\text { e. Ri eye }=87 \mathrm{cGy} \\
\text { r. Lt eye }=69 \mathrm{cGy} \\
\text { g. Spinal cord }=130 \mathrm{cGy}(\mathrm{Max}) \\
\text { h. Cerebellum }=3316 \mathrm{cGy}\end{array}$ & $\begin{array}{l}\text { Organ At Risk (OAR) dose: } \\
\text { a. Rt optic nerve }=139 \mathrm{cGy} \\
\text { b. Lt oplic nerve }=97 \mathrm{cGy} \\
\text { c. Rt eyc lens }=73 \mathrm{cGy} \\
\text { d. LL eyc lens }=53 \mathrm{cGy} \\
\text { e. Rt eye }=91 \mathrm{cGy} \\
\text { C. Lt eye }=64 \mathrm{cGy} \\
\text { g. Spinal cord }=109 \mathrm{cGy}(\mathrm{Max}) \\
\text { h. Cerebellum = } 593 \mathrm{cGy}\end{array}$ \\
\hline $\begin{array}{l}\text { Homogeneity Indices: } \\
\begin{aligned} H I & =\frac{\mathrm{D} 2-\mathrm{D} 98}{\mathrm{DP}} \times 100 \% \\
& =6.6\end{aligned}\end{array}$ & $\begin{array}{l}\text { Homogeneity Indices: } \\
\begin{aligned} H A & =\frac{D 2-D 98}{D P} \times 100 \% \\
& =4.8\end{aligned}\end{array}$ \\
\hline $\begin{array}{l}\text { Conformity Index: } \\
\begin{aligned} & c x(95 \%)=V(95 \%) \\
& V(\text { CPTV }\end{aligned} \\
=0.99\end{array}$ & $\begin{array}{l}\text { Conformity Index: } \\
\begin{aligned} \mid c r(95 \%) & =\frac{V(95 \%)}{V(P T V)} \\
& =0.99\end{aligned}\end{array}$ \\
\hline
\end{tabular}




\section{CONCLUSION}

This case study refers to a female patient of age 42 of Dhaka. Diagnosis was Glioblastoma Multiforme (GBM) stage IV. The patient is a typical example of such clinical cases where decisions are required for a treatment of choice. Table 1 highlights the fact that IMRT is a better option for to treat such a case. Comparable dose values for each anatomy show a lower dose to the OARs and, for that matter, "cerebellum". Figs $4 \mathrm{a}$ and $4 \mathrm{~b}$ show the DVH where delivered dose in two plans shows a better precision in IMRT. Similarly, differential DVH curves in Figs $5 \mathrm{a}$ and $5 \mathrm{~b}$ depict a better accuracy of dose per volume at the prescribed dose in IMRT plan. The relevant parameters are evaluated before approving a plan to treat a cancer. If, for some reason, there is a great anomaly in some values like hot and cold spots or those mentioned in section 2.2.4 and table 1, the treatment plan is to be redone using all treatment tools to shape the beam to conform the dose to tumor volume. Accepted plan can only be deliverable for quality treatment.

\section{ACKNOWLEDGMENT}

Our sincere thanks are due to Professor S. Mukarram Ali, FRC Path, in providing the facilities for such a treatment, using a multi-energy LINAC for radiation treatment.

\section{REFERENCES}

1. International Commission on Radiation Units and Measurements I (1993) ICRU Report 50, prescribing, recording, and reporting photon beam therapy. Nuclear Technology Publishing, Bethesda, MD, USA.

2. International Commission on Radiation Units and Measurements I (1999) ICRU report 62: prescribing, recording and reporting photon beam therapy (supplement to ICRU report 50). Nuclear Technology Publishing, Bethesda, MD, USA.

3. Withers, H. R., and Thames, H. D.. "Dose fractionation and volume effects in normal tissues and tumor, "Am. J. Clin. Oncol 1988. 11, 313-329.

4. Emami B, Lyman J, Brown A et al.Tolerance of normal tissue to therapeutic irradiation. Int $\mathrm{J}$ Radiat Oncol Biol Phys 199121:109-122.

5. Spetzler RF, Martin NA, A proposed grading system arteriovenous malformations, J Neurosurg 1986; 65:476-483.

6. Bottomley, P. A., Foster, T. H., Argersinger, R. E., et al. A review of normal tissue hydrogen NMR relaxation times and relaxation mechanisms from 1-100 MHz: Dependence on tissue type, NMR frequency, temperature, species, excision, and age. Med Phys 1984; 11:425-448.

7. Laperriere N, Zuraw L, Cairncross G .The Cancer Care Ontario Practice Guidelines Initiative Neuro-Oncology Disease Site Group. Radiotherapy for newly diagnosed malignant glioma in adults: a systematic review. Radiother Oncol 2002;64: 259-73.

8. Shawl EG, Seiferheld W, Scott C et al.Re-examining the radiation therapy oncology group (RTOG) recursive partitioning analysis (RPA) for glioblastoma multiform (GBM) patients. Radiat Oncol Biol Phys 200357: S135-6. 